Supplement of

\title{
Ozone deposition to a coastal sea: comparison of eddy covariance observations with reactive air-sea exchange models
}

David C. Loades et al.

Correspondence to: David C. Loades (d1823@york.ac.uk)

The copyright of individual parts of the supplement might differ from the CC BY 4.0 License. 


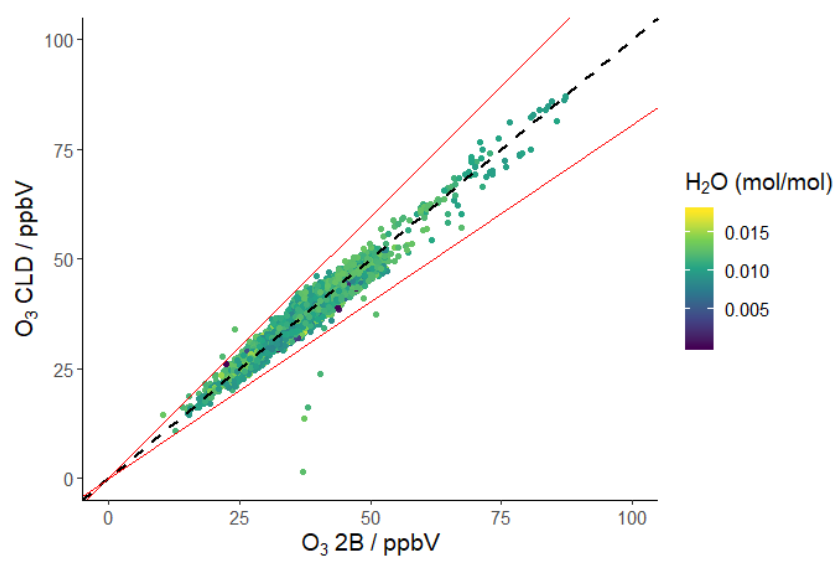

Figure S1: Comparison of 2B and CLD measured ozone concentration using a fixed sensitivity for the CLD of 240 counts $\operatorname{ppbv}^{-1} \mathrm{~s}^{-1}$. The dotted line is $x=y$, and the red lines indicate a $3 \sigma$ deviation from mean sensitivity, used in data filtering. Colour bar indicates the water vapour mixing ratio. Humidity does not appear to majorly affect sensitivity.

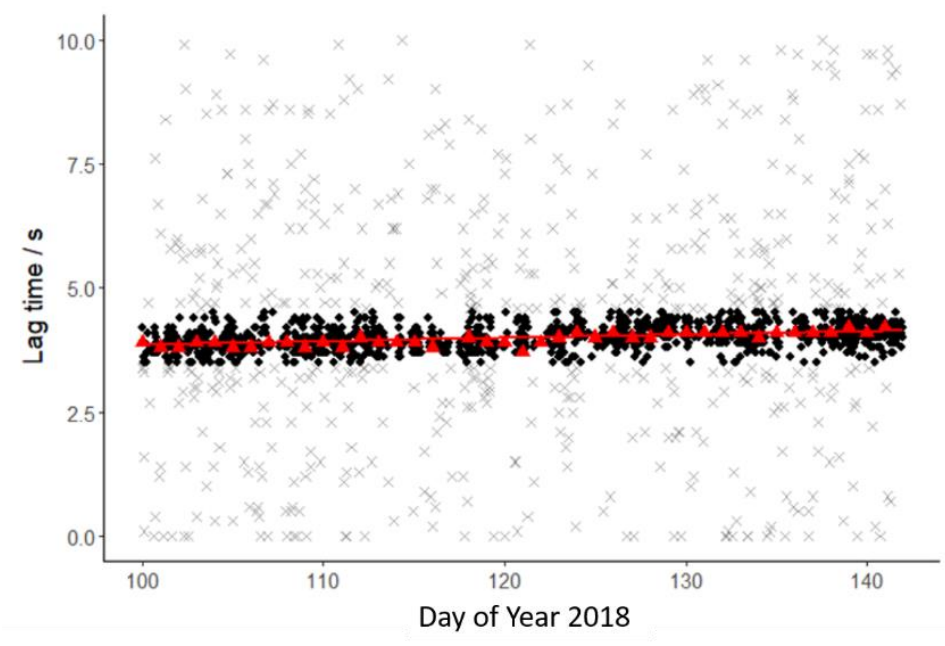

Figure S2: Lag times determined for each 20-minute period. Lags between 3.5 and 4.5 seconds (black dots) were accepted and used to plot a linear fit (red line). Determined lags outside of these bounds (grey crosses) were rejected, and were instead set to the linear fit. Lags determined from daily CCF are shown as red triangles. 


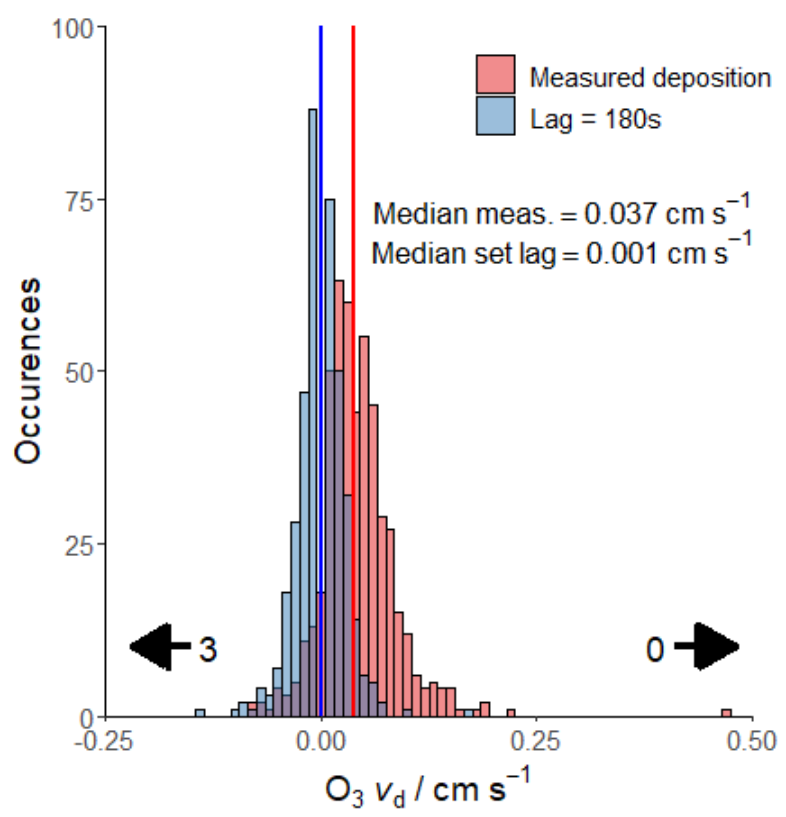

Figure S3: Observed deposition velocity (red) vs deposition observed with lag $=180 \mathrm{~s}$ (blue). Medians given by respectively coloured lines. $\mathrm{X}$ axis limited from $\mathbf{- 0 . 2 5}-\mathbf{0 . 5}$ for clarity, with the number of points out of these bound indicated by the arrows. The $180 \mathrm{~s}$ lag values serve as a measure of noise in the measurements, and a Kolmogorov-Smirnov test suggests our measured values could not have come from this noise by chance. 